ALEA, Lat. Am. J. Probab. Math. Stat. 15, 837-849 (2018)

DOI: 10.30757/ALEA.v15-31

\title{
First Passage Time Densities through Hölder curves
}

\section{Jimyeong Lee}

Gran Sasso Science Institute, Viale Francesco Crispi, 7, 67100, L'Aquila, Italy

E-mail address: jimyeong.lee@gssi.infn.it

\begin{abstract}
We prove that for a standard Brownian motion, there exists a firstpassage-time density function through a Hölder curve with exponent greater than $1 / 2$. With a property of local time of a standard Brownian motion and adopting the theories of partial differential equations in Cannon (1984) and the strategies in Fasano (2005) and Carinci et al. (2016), we find a sufficient condition for existence of the density function. We also show that this density function is proportional to the space derivative of the Green function of the heat equation with Dirichlet boundary condition at the moving boundary.
\end{abstract}

\section{Introduction}

In this paper we will study the probability of the hitting time to a moving boundary. To state the main result, we need some notations on a Brownian motion. Let us call $P_{r, s}, r \in \mathbb{R}, s \geq 0$, the law on $C([s, \infty))$ of the Brownian motion $B_{t}, t \geq s$, which starts from $r$ at time $s$, i.e. $B_{s}=r$. For each $t>s$ the law of $B_{t}$ is absolutely continuous with respect to the Lebesgue measure and has a density $G_{s, t}(r, \cdot)$ which is the Gaussian $G(\cdot, t ; r, s)=\frac{1}{\sqrt{2 \pi(t-s)}} \exp \left(-\frac{(\cdot-r)^{2}}{2(t-s)}\right)$. We denote by $E_{r, s}$ the expectation under $P_{r, s}$. For a given curve $X=\left\{t \rightarrow X_{t}\right\}, s \geq 0$ and $r<X_{s}$, we define

$$
\tau_{r, s}^{X}=\inf \left\{t \geq s: B_{t} \geq X_{t}\right\}, \text { and }=\infty \text { if the set is empty, }
$$

where $B_{s}=r$ and denote by $F_{r, s}^{X}(d t)$ the distribution of $\tau_{r, s}^{X}$ induced by $P_{r, s}$. For $\mathrm{s}=0$, we use abbreviated forms $P_{r}, E_{r}, \tau_{r}^{X}, F_{r}^{X}(d x)$ instead of $P_{r, 0}, E_{r, 0}, \tau_{r, 0}^{X}$, $F_{r, 0}^{X}(d x)$ respectively whenever it is needed. In addition, for $t>0$, let us call $d \mu_{r_{0}}(\cdot, t)$ the positive measure on $\left(-\infty, X_{t}\right)$ such that

$$
\int_{\left(-\infty, X_{t}\right)} d \mu_{r_{0}}(x, t) f(x)=E_{r_{0}}\left[f\left(B_{t}\right) ; \tau_{r_{0}}^{X}>t\right]
$$

Received by the editors November 8th, 2017; accepted July 10th, 2018.

2010 Mathematics Subject Classification. 60G99, 35K05.

Key words and phrases. First Passage Time Densities, Brownian motion, Heat equation. 
for all $f \in C_{c}^{\infty}(\mathbb{R})$ with supp $f \Subset\left(-\infty, X_{t}\right)$. The main result in the paper is:

Theorem 1.1. If $X$ is Hölder continuous on any finite interval in $[0, \infty)$ with exponent $\gamma \in(1 / 2,1]$ and $r_{0}<X_{0}$, then

(1) $d \mu_{r_{0}}(x, t)=G_{0, t}^{X}\left(r_{0}, x\right) d x$ where for all $x<X_{t}$,

$$
G_{0, t}^{X}\left(r_{0}, x\right)=G_{0, t}\left(r_{0}, x\right)-\int_{[0, t)} F_{r_{0}}^{X}(d s) G_{s, t}\left(X_{s}, x\right) .
$$

(2) $F_{r_{0}}^{X}(d s)$ has a density function $p$ on $[0, \infty)$, namely $F_{r_{0}}^{X}(d s)=p(s) d s$.

(3) $p(t)=-\left.\frac{1}{2} \frac{\partial}{\partial x} G_{0, t}^{X}\left(r_{0}, x\right)\right|_{x=X_{t}^{-}}$for all $t>0$.

(4) $G_{0, t}^{X}\left(r_{0}, x\right)$ solves

$$
\begin{array}{r}
v_{t}=\frac{1}{2} v_{x x}, \quad-\infty<x<X_{t}, t>0, \\
\lim _{(x, t) \rightarrow\left(X_{s}, s\right)} v(x, t)=0, s>0, \\
\lim _{(x, t) \rightarrow(y, 0)} v(x, t)=\delta_{r_{0}}(y),-\infty<y<X_{0} .
\end{array}
$$

\section{Remarks}

Item 4 of Theorem 1.1 states that for any $r_{0}<X_{0}$ the function $G_{0, \cdot}^{X}\left(r_{0}, \cdot\right)$ given by (1.3) is the Green function of the heat equation with Dirichlet boundary conditions at the moving boundary $X$. In Alili and Patie (2014), when a moving boundary is infinitely differentiable, it is showed that the space derivative of the Green function of the heat equation at the boundary is proportional to the hitting time density function. Likewise by items 2 and 3 of Theorem 1.1, the space derivative of $G_{0, \cdot}^{X}\left(r_{0}, \cdot\right)$ at the moving boundary is proportional to the hitting time density function $p$.

For the case when $X_{t}=a+b t$, it is well known(see for instance Karatzas and Shreve, 1991) that for $r<a, \tau_{r}^{X}$ has a probability density function given by $f(t)=$ $\frac{a-r}{\sqrt{2 \pi t^{3}}} \exp \left(-\frac{(a+b t-r)^{2}}{2 t}\right) \mathbf{1}_{t>0}$. In addition, there is another result in Ricciardi et al. (1984) when $X_{t}=a+b t^{\frac{1}{p}}$ for $p \geq 2, r<a$, then $\tau_{r}^{X}$ has a probability density function. In Borovkov and Downes (2010), it is showed that if the boundary behaves as a Lipschitz curve in a local time, then the first passage time density can be expressed explicitly.

In Peskir and Shiryaev (2006), it is proved that for any continuous curve $X_{t}$ and $r<X_{0}$, there is a distribution $F_{r}^{X}$ of $\tau_{r}^{X}$ which satisfies the following integral equation(called the Master Equation):

$$
\Psi\left(\frac{z-r}{\sqrt{t}}\right)=\int_{0}^{t} \Psi\left(\frac{z-X_{s}}{\sqrt{t-s}}\right) F_{r}^{X}(d s),
$$

where $z \geq X_{t}, t>0$ and $\Psi(z)=\int_{z}^{\infty} \frac{1}{\sqrt{2 \pi}} \exp \left(-\frac{x^{2}}{2}\right) d x$. This can be proved intuitively as follows; the left hand side of (1.7) is the probability that a Brownian motion starts at $r$ at time 0 and reaches $z$ greater or equal to $X_{t}$ at time $t$. Then it should hit the boundary at least once which implies the right hand side of of (1.7). Moreover, it is showed that if $X_{t}$ is $C^{1}$, then there exists a continuous density 
function $f$ of $F_{r}^{X}$. There is an extension of this result, in Ricciardi et al. (1984), to curves $X$ which are differentiable with $\left|\frac{d X_{t}}{d t}\right| \leq C t^{-\alpha}$ for some constant $C>0$ and $\alpha<1 / 2$.

By (1.7), for any continuous curve $X_{t}$ and $r<X_{0}$, we have

$$
\Psi\left(\frac{X_{t}-r}{\sqrt{t}}\right)=\int_{0}^{t} \Psi\left(\frac{X_{t}-X_{s}}{\sqrt{t-s}}\right) F_{r}^{X}(d s) .
$$

which can be regarded as an integral equation for $F_{r}^{X}(d s)$. In Taillefumier and Magnasco (2014), the equation (1.8) is studied when $X$ is Hölder continuous with exponent greater than $1 / 2$. It is proved that there exists a unique continuous function $q$ such that

$$
\Psi\left(\frac{X_{t}-r}{\sqrt{t}}\right)=\int_{0}^{t} \Psi\left(\frac{X_{t}-X_{s}}{\sqrt{t-s}}\right) q(s) d s .
$$

To conclude that $F_{r}^{X}(d s)=q(s) d s$, one still needs that $F_{r}^{X}(d s)$ is absolutely continuous with respect to Lebesgue measure. The analysis in Taillefumier and Magnasco (2014) as well as the proof of Theorem 1.1 uses extensively the work by Cannon Cannon (1984) on the heat equation with the moving boundary.

\section{Preliminaries: a weaker form of Theorem 1.1}

We define $D$ as

$$
D:=\left\{(x, t):-\infty<x<X_{t}, t>0\right\}
$$

and

$$
u(x, t):=\int_{-\infty}^{0} h(\xi) G_{0, t}^{X}(\xi, x) d \xi
$$

for given $h \in C_{c}^{\infty}\left(\left(-\infty, X_{0}\right) ; \mathbb{R}_{+}\right)$and all $(x, t) \in D$.

If $X$ is Hölder continuous with exponent $\gamma \in[1 / 2,1]$, then $u$ solves the heat equation in $D$ with the initial condition $h$ and Dirichlet boundary condition.(See Theorem 2.1 below) However uniqueness fails for the heat equation with the initial function $h$ and Dirichlet boundary conditions (see Remark 2.2 and 2.3 below).

To have the uniqueness, first of all, we restrict time variable of $D$ in a finite interval. Thus we fix $T>0$ and define the parabolic cylinder $D_{T}$ which is a subset of $D$ as

$$
D_{T}:=\left\{(x, t):-\infty<x<X_{t}, 0<t \leq T\right\} .
$$

Consider the following initial-boundary value problem

$$
\left\{\begin{array}{l}
v \in C\left(\overline{D_{T}}\right) \cap C^{2,1}\left(D_{T}\right), \\
v_{t}=\frac{1}{2} v_{x x}, \quad(x, t) \in D_{T}, \\
v\left(X_{t}, t\right)=0,0<t \leq T, \\
v(x, 0)=h(x),-\infty<x<X_{0}, \\
\lim _{x \rightarrow-\infty} \sup _{0<t<T}|v(x, t)|=0 .
\end{array}\right.
$$

We prove the following weaker form of Theorem 1.1.

Theorem 2.1. Let $X$ be a Hölder continuous curve on any finite interval in $[0, \infty)$ with exponent $\gamma \in[1 / 2,1]$ and let $X_{0}=0$. 
(1) The function $u$ defined in (2.1) is the unique solution of (2.2).

(2) If $\gamma \in(1 / 2,1]$, then $u$ has the left hand derivative at the boundary $u_{x}\left(X_{t}^{-}, t\right)$ which is continuous on $(0, \infty)$. Moreover, for all $t>0, p_{h}(t):=$ $-\frac{1}{2} u_{x}\left(X_{t}^{-}, t\right)$ satisfies

$$
p_{h}(t)=-\int_{-\infty}^{0} h(\xi) G_{x}\left(X_{t}, t ; \xi, 0\right) d \xi+\int_{0}^{t} G_{x}\left(X_{t}, t ; X_{\tau}, \tau\right) p_{h}(\tau) d \tau .
$$

Remark 2.2. The uniqueness for (2.2) is not guaranteed if we do not assume $v \in C\left(\overline{D_{T}}\right)$. When $X_{t}=0$ for all $t$ and $h$ is identically 0 , if $v(x, t)$ is given by $\frac{1}{\sqrt{2 \pi t}}\left\{-\frac{x}{t}\right\} \exp \left(-\frac{x^{2}}{2 t}\right)$, then this satisfies the heat equation with the initial data 0 and is also 0 on the boundary, but this is not continuous at $(0,0)$.

Remark 2.3. We need the condition $\lim _{x \rightarrow-\infty} \sup _{0<t<T}|v(x, t)|=0$ to have uniqueness. Indeed, for $X_{t}=0$ for all $t \geq 0$, the function

$$
v(x, t)=\sum_{n=0}^{\infty} f^{(n)}\left(\frac{t}{2}\right) \frac{x^{2 n+1}}{(2 n+1) !}
$$

where

$$
f(t)=\left\{\begin{array}{l}
\exp \left(-\frac{1}{t^{2}}\right), \quad t>0 \\
0, \quad t \leq 0
\end{array}\right.
$$

satisfies the heat equation with the initial data 0 and is also 0 on the boundary. Furthermore, $v \in C\left(\overline{D_{T}}\right) \cap C^{2,1}\left(D_{T}\right)$.

Remark 2.4. It can be shown $\lim _{t \rightarrow 0} p_{h}(t)=0$ by (2.3) and Lemma 3.3 below. Thus $u_{x}\left(X_{t}^{-}, t\right)$ is continuous on $[0, \infty)$.

Following Cannon (1984), we introduce $C_{(\nu)}^{0}((0, T]), 0<\nu \leq 1$, as the subspace of $C((0, T])$ that consists of those functions $\varphi$ such that

$$
\|\varphi\|_{T}^{(\nu)}=\sup _{0<t \leq T} t^{1-\nu}|\varphi(t)|<\infty .
$$

Then $C_{(\nu)}^{0}((0, T])$ is a Banach space under the norm $\|\cdot\|_{T}^{(\nu)}$.

We also introduce the following lemmas from Cannon (1984) which play an essential role in our analysis.

Lemma 2.5 (jump relation). For $\varphi \in C_{(\nu)}^{0}((0, T])$, we have

$$
\lim _{x \rightarrow X_{t}^{ \pm}} \frac{\partial w_{\varphi}}{\partial x}(x, t)=\mp \varphi(t)+\int_{0}^{t} G_{x}\left(X_{t}, t ; X_{\tau}, \tau\right) \varphi(\tau) d \tau,
$$

where $w_{\varphi}(x, t)=\int_{0}^{t} G\left(x, t ; X_{\tau}, \tau\right) \varphi(\tau) d \tau$.

For two continuous curves $s_{1}, s_{2}$ such that $s_{1}(t)<s_{2}(t), t \in[0, T]$, let us set $E_{T}:=\left\{(x, t): s_{1}(t)<x<s_{2}(t), 0<t \leq T\right\}$ and $B_{T}:=\left\{\left(s_{i}(t), t\right): 0 \leq t \leq T, i \in\right.$ $\{1,2\}\} \cup\left\{(x, 0): s_{1}(0)<x<s_{2}(0)\right\}$. 
Lemma 2.6 (The Weak Maximum(Minimum) Principle). For a solution $u$ of $u_{t}=$ $\frac{1}{2} u_{x x}$ in $E_{T}$, which is continuous in $E_{T} \cup B_{T}$,

$$
\max _{E_{T} \cup B_{T}} u=\max _{B_{T}} u . \quad\left(\min _{E_{T} \cup B_{T}} u=\min _{B_{T}} u .\right)
$$

Before going to the proof of Theorem 2.1, we need the following proposition.

Proposition 2.7. Let $X$ be a Hölder continuous curve on any finite interval in $[0, \infty)$ with exponent $\gamma \in[1 / 2,1]$. If the starting point of the Brownian motion is close to $X$, the first hitting time converges to 0. Precisely, $\lim _{\xi \rightarrow X_{0}} P_{\xi}\left[\tau_{\xi}^{X}>s\right]=0$ for all $s>0$.

Proof: Without loss of generality, we may reduce this problem as the case for Brownian motion starting at 0 and $X_{0}=\epsilon>0$ and let $\epsilon$ go to 0 . For $s>0$, let $m:=\sup _{0 \leq t_{1}<t_{2} \leq s} \frac{\left|X_{t_{2}}-X_{t_{1}}\right|}{\left|t_{2}-t_{1}\right|^{\gamma}}$. Since $\limsup _{t \downarrow 0} \frac{B_{t}}{\sqrt{t}}=\infty$ a.e., for $M>m$, we have a sequence $t_{k} \downarrow 0$ such that $M \sqrt{t_{k}} \leq B_{t_{k}}$ a.e. and $M \sqrt{t_{k}}-m t_{k}^{\gamma} \downarrow 0$ for all $k$. Thus, for $0<t \leq s$, we deduce that a.e.

$$
\begin{aligned}
\sup _{0 \leq l \leq t}\left\{B_{l}-X_{l}\right\} \geq \sup _{0 \leq l \leq t}\left\{B_{l}-\left(\epsilon+m l^{\gamma}\right)\right\} & \geq \sup _{t_{k} \leq t}\left\{B_{t_{k}}-\left(\epsilon+m t_{k}^{\gamma}\right)\right\} \\
& \geq \sup _{t_{k} \leq t}\left\{M \sqrt{t_{k}}-\left(\epsilon+m t_{k}^{\gamma}\right)\right\} .
\end{aligned}
$$

Therefore,

$$
P_{0}\left(\sup _{0 \leq l \leq t}\left\{B_{l}-X_{l}\right\}<0\right) \leq P_{0}\left(\sup _{t_{k} \leq t}\left\{M \sqrt{t_{k}}-m t_{k}^{\gamma}\right\}<\epsilon\right) .
$$

For each sufficently small $\epsilon>0$, there is a greatest $k(\epsilon)$ such that $M \sqrt{t_{k(\epsilon)}}-$ $m t_{k(\epsilon)}^{\gamma} \geq \epsilon$. Thus we obtain that $\tau_{0}^{X} \leq t_{k(\epsilon)}$ a.e. for all sufficiently small $\epsilon>0$. Since $k(\epsilon)$ is an increasing function as $\epsilon$ decreases and $\lim _{\epsilon \rightarrow 0} k(\epsilon)=\infty$, the proposition follows.

Proof of Theorem 2.1: Let us define $X_{\tau}^{\prime}:=X_{t-\tau}$ for all $0 \leq \tau \leq t$. Using the invariance of the law of the Brownian motion under time reversal, we have

$$
u(x, t)=\int_{-\infty}^{0} h\left(r^{\prime}\right) G_{0, t}^{X}\left(r^{\prime}, x\right) d r^{\prime}=E_{x}\left[h\left(B_{t}\right) ; \tau_{x}^{X^{\prime}}>t\right] .
$$

Using this equality, we also have

$$
|u(x, t)|=\left|E_{x}\left[h\left(B_{t}\right) ; \tau_{x}^{X^{\prime}}>t\right]\right| \leq\|h\|_{\infty} P_{x}\left[\tau_{x}^{X^{\prime}}>t\right] .
$$

For $s>0$, let us choose $0<s^{*}<s$. Then for all $(x, t)$ sufficiently close to $\left(X_{s}, s\right)$, we obtain

$$
P_{x}\left[\tau_{x}^{X^{\prime}}>t\right] \leq P_{x}\left[\tau_{x}^{X^{\prime}}>s^{*}\right]
$$

which vanishes when $(x, t) \rightarrow\left(X_{s}, s\right)$ by Proposition 2.7 so that $\lim _{(x, t) \rightarrow\left(X_{s}, s\right)} u(x, t)=$ 0 . In addition, we have

$$
|u(x, t)|=\left|E_{x}\left[h\left(B_{t}\right) ; \tau_{x}^{X^{\prime}}>t\right]\right| \leq E_{x}\left[\left|h\left(B_{t}\right)\right|\right]=\int_{-\infty}^{0}|h(\xi)| G_{0, t}(x, \xi) d \xi
$$


which also vanishes when $(x, t) \rightarrow(0,0)$, since the support of $h$ is strictly away from 0 . To prove that $u$ satisfies the initial data $h$, we write $y_{t}=\min _{s \in[0, t]} X_{s}^{\prime}$. For $x<0$ and any positive $\lambda>0$,

$$
P_{x}\left[\tau_{x}^{X^{\prime}} \leq t\right] \leq P_{x}\left[\max _{s \in[0, t]} B_{s} \geq y_{t}\right]=P_{x}\left[\max _{s \in[0, t]} \exp \left(\lambda B_{s}\right) \geq \exp \left(\lambda y_{t}\right)\right] .
$$

Since the exponential of Brownian motion is a positive submartingale, we can apply Doob's inequality, then

$P_{x}\left[\max _{s \in[0, t]} \exp \left(\lambda B_{s}\right) \geq \exp \left(\lambda y_{t}\right)\right] \leq \frac{E_{x}\left[\exp \left(\lambda B_{t}\right)\right]}{\exp \left(\lambda y_{t}\right)}=\exp \left(\frac{1}{2} \lambda^{2} t-\lambda\left(y_{t}-x\right)\right)$.

From (2.10) and (2.11), we get

$$
\lim _{(x, t) \rightarrow(y, 0)} P_{x}\left[\tau_{x}^{X^{\prime}} \leq t\right] \leq \exp \left(-\lambda\left(X_{0}-y\right)\right)=\exp (\lambda y)
$$

so that the left hand side vanishes since $\lambda>0$ is arbitrary and we are assuming $y<X_{0}=0$. Thus we obtain that

$$
\lim _{(x, t) \rightarrow(y, 0)} \int_{-\infty}^{0} h(\xi) G_{0, t}^{X}(\xi, x) d \xi=\lim _{(x, t) \rightarrow(y, 0)} E_{x}\left[h\left(B_{t}\right)\right]=h(y) .
$$

By the properties of the Gaussian kernel, we deduce that $u$ solves (2.2). We now prove uniqueness. If $v_{1}, v_{2}$ satisfy all the above conditions, then $v_{1}-v_{2} \in C\left(\overline{D_{T}}\right) \cap$ $C^{2,1}\left(D_{T}\right)$ satisfies the heat equation with the initial data 0 and is also 0 on the boundary. Moreover, $\lim _{x \rightarrow-\infty} \sup _{0<t<T}\left|v_{1}(x, t)-v_{2}(x, t)\right|=0$ so that by the weak maximum(minimum) principle, $v_{1}-v_{2}$ is all 0 in $D_{T}$. Therefore, $v_{1}=v_{2}$ so that item 1 is proved.

To prove item 2, assuming that $\gamma \in(1 / 2,1]$, we define, $(x, t) \in D_{T}$,

$$
v(x, t):=\int_{-\infty}^{0} h(\xi) G(x, t ; \xi, 0) d \xi+\int_{0}^{t} G\left(x, t ; X_{\tau}, \tau\right) \varphi(\tau) d \tau,
$$

where $\varphi \in C_{(\gamma)}^{0}((0, T])$ satisfies

$$
0=\int_{-\infty}^{0} h(\xi) G\left(X_{t}, t ; \xi, 0\right) d \xi+\int_{0}^{t} G\left(X_{t}, t ; X_{\tau}, \tau\right) \varphi(\tau) d \tau .
$$

If we apply the Abel inverse operator $A$ defined by

$$
(A F)(t)=\frac{1}{\pi} \frac{d}{d t} \int_{0}^{t} \frac{F(\eta)}{(t-\eta)^{\frac{1}{2}}} d \eta
$$

on both sides of (2.15), then from Chapter 14 in Cannon (1984) we have a equivalent Volterra integral equation of the second kind:

$$
\varphi(t)=\psi(t)+\int_{0}^{t} H(t, \tau) \varphi(\tau) d \tau
$$

where $\psi \in C_{(\gamma)}^{0}((0, T])$ and $|H(t, \tau)| \leq C(t-\tau)^{2 \gamma-2}$. The existence of $\varphi \in$ $C_{(\gamma)}^{0}((0, T])$ which satisfies $(2.16)$ can be proved similarly to the proof of Proposition 3.2 that we will show later. Then $v$ is well-defined and solves (2.2) so that 
$v=u$ since $u$ is the unique solution of (2.2). By the jump relation, we have

$$
u_{x}\left(X_{t}^{-}, t\right)=\int_{-\infty}^{0} h(\xi) G_{x}\left(X_{t}, t ; \xi, 0\right) d \xi+\varphi(t)+\int_{0}^{t} G_{x}\left(X_{t}, t ; X_{\tau}, \tau\right) \varphi(\tau) d \tau
$$

so that $u_{x}\left(X_{t}^{-}, t\right) \in C_{(\gamma)}^{0}((0, T])$. Since $T$ is arbitrary, we deduce $u_{x}\left(X_{t}^{-}, t\right)$ is continuous on $(0, \infty)$.

To show (2.3), let us fix $(x, t) \in D_{T}$ and let us define $D_{\epsilon, r}^{(t)}:=\{(\xi, \tau): r<\xi<$ $\left.X_{\tau}-\epsilon, \epsilon<\tau<t-\epsilon\right\}$ for each $\epsilon>0$ and $r \in \mathbb{R}$. By the Green's identity, we have

$$
\frac{1}{2}\left(u_{\xi} G-u G_{\xi}\right)_{\xi}-(u G)_{\tau}=0 \Longrightarrow \oint_{\partial D_{\epsilon, r}^{(t)}} \frac{1}{2}\left(u_{\xi} G-u G_{\xi}\right) d \tau+(u G) d \xi=0
$$

It can be also shown that $\lim _{x \rightarrow-\infty} \sup _{0<t<T}\left|u_{x}(x, t)\right|=0$ by the properties of the Gaussian kernel. Hence we obtain another representation of $u$ by letting $\epsilon \rightarrow 0, r \rightarrow-\infty$,

$$
u(x, t)=\int_{-\infty}^{0} h(\xi) G(x, t ; \xi, 0) d \xi+\frac{1}{2} \int_{0}^{t} G\left(x, t ; X_{\tau}, \tau\right) u_{x}\left(X_{\tau}^{-}, \tau\right) d \tau .
$$

Differentiating both sides of (2.19) with respect to $\mathrm{x}$ and applying the jump relation, we get

$$
\frac{1}{2} u_{x}\left(X_{t}^{-}, t\right)=\int_{-\infty}^{0} h(\xi) G_{x}\left(X_{t}, t ; \xi, 0\right) d \xi+\frac{1}{2} \int_{0}^{t} G_{x}\left(X_{t}, t ; X_{\tau}, \tau\right) u_{x}\left(X_{\tau}^{-}, \tau\right) d \tau .
$$

which implies (2.3).

\section{Proof of Theorem 1.1}

To prove item 1 , let $X$ be a continuous curve defined on $[0, \infty)$ and let $r_{0}<X_{0}$. Using the strong Markov property, we have, for $f \in C_{c}^{\infty}(\mathbb{R})$ with supp $f \Subset\left(-\infty, X_{t}\right)$ and $0 \leq s \leq t$,

$$
E_{r_{0}}\left[f\left(B_{t}\right) \mid \tau_{r_{0}}^{X}=s\right]=E_{X_{s}, s}\left[f\left(B_{t}\right)\right]
$$

Thus we get

$$
E_{r_{0}}\left[f\left(B_{t}\right) ; \tau_{r_{0}}^{X} \leq t\right]=\int_{[0, t]} F_{r_{0}}^{X}(d s) E_{X_{s}, s}\left[f\left(B_{t}\right)\right] .
$$

It follows that item 1 holds with $G_{0, t}^{X}\left(r_{0}, x\right)$ given by (1.3).

To prove item 2, from now on, $X$ is a Hölder continuous curve defined on $[0, \infty)$ with exponent $\gamma \in(1 / 2,1]$ and $X_{0}=0$. Comparing the definition (2.1) of $u$ and (2.19), using (1.3), we see the following equality:

$$
\int_{[0, t)} G_{\tau, t}\left(X_{\tau}, x\right) \int_{-\infty}^{0} h(\xi) F_{\xi}^{X}(d \tau) d \xi=-\frac{1}{2} \int_{0}^{t} G\left(x, t ; X_{\tau}, \tau\right) u_{x}\left(X_{\tau}^{-}, \tau\right) d \tau .
$$

Set $F_{h}^{X}(d \tau):=\int_{-\infty}^{0} h(\xi) F_{\xi}^{X}(d \tau) d \xi$

Proposition 3.1. $F_{h}^{X}(d \tau)=-\frac{1}{2} u_{x}\left(X_{\tau}^{-}, \tau\right) d \tau$. 
For the proof of Proposition 3.1, we introduce the mass lost $\Delta_{I}^{X}(u), I=\left[t_{1}, t_{2}\right] \subset$ $[0, T], t_{1} \leq t_{2}$, is defined by

$$
\Delta_{I}^{X}(u)=\int_{-\infty}^{X_{t_{1}}} u\left(r, t_{1}\right) d r-\int_{-\infty}^{X_{t_{2}}} u\left(r, t_{2}\right) d r .
$$

If we see the right hand side of (2.19), we can extend $u$ to $\bar{u}$ defined in $\{(x, t): x \in$ $\mathbb{R}, 0<t \leq T\}$ as

$$
\bar{u}(x, t)=\int_{-\infty}^{0} h(\xi) G(x, t ; \xi, 0) d \xi+\frac{1}{2} \int_{0}^{t} G\left(x, t ; X_{\tau}, \tau\right) u_{x}\left(X_{\tau}^{-}, \tau\right) d \tau .
$$

Then this satisfies the heat equation with $\lim _{(x, t) \rightarrow(y, 0)} \bar{u}(x, t)=0$ for all $y \geq 0$ and also satisfies $\bar{u}\left(X_{t}, t\right)=0$ for all $0<t \leq T$. Moreover, by the properties of Gaussian kernel, we have

$$
\lim _{x \rightarrow \infty} \sup _{0<t<T}|\bar{u}(x, t)|=0 .
$$

It follows that $\bar{u}(x, t)=0$ in $\left\{(x, t): x \geq X_{t}, 0<t \leq T\right\}$ by the weak maximum(minimum) principle. Thus we assume that $u$ is defined $\{(x, t): x \in \mathbb{R}, 0 \leq$ $t \leq T\}$ such that it is 0 in $\left\{(x, t): x \geq X_{t}, 0 \leq t \leq T\right\}$.

Heuristically

$$
\Delta_{I}^{X}(u)=-\iint_{t_{1}}^{t_{2}} u_{t}(x, t) d t d x=-\iint_{t_{1}}^{t_{2}} \frac{1}{2} u_{x x}(x, t) d x d t=-\frac{1}{2} \int_{t_{1}}^{t_{2}} u_{x}\left(X_{t}^{-}, t\right) d t .
$$

Since we do not control $u_{x x}$ at the moving boundary, we cannot make this argument rigorously. Thus we use a different approach.

Proof of Proposition 3.1: It suffices to show

$$
-\frac{1}{2} \int_{I} u_{x}\left(X_{t}^{-}, t\right) d t=\Delta_{I}^{X}(u)=F_{h}^{X}(I) .
$$

If we integrate both sides of (2.19), then

$$
\begin{aligned}
\int_{-\infty}^{\infty} u(x, t) d x= & \int_{-\infty}^{\infty} \int_{-\infty}^{0} h(\xi) G(x, t ; \xi, 0) d \xi d x \\
& +\int_{-\infty}^{\infty} \frac{1}{2} \int_{0}^{t} G\left(x, t ; X_{\tau}, \tau\right) u_{x}\left(X_{\tau}^{-}, \tau\right) d \tau d x
\end{aligned}
$$

Applying Fubini's theorem, we get

$$
\int_{-\infty}^{X_{t}} u(x, t) d x=\int_{-\infty}^{\infty} u(x, t) d x=\int_{-\infty}^{0} h(\xi) d \xi+\frac{1}{2} \int_{0}^{t} u_{x}\left(X_{\tau}^{-}, \tau\right) d \tau .
$$

Thus we get the first equality of the proposition,

$$
\Delta_{\left[t_{1}, t_{2}\right]}^{X}(u)=-\frac{1}{2} \int_{t_{1}}^{t_{2}} u_{x}\left(X_{\tau}^{-}, \tau\right) d \tau .
$$

From (1.2) and item 1 of Theorem 1.1, we get

$$
P_{\xi}\left[\tau_{\xi}^{X}>t\right]=\int_{-\infty}^{X_{t}} G_{0, t}^{X}(\xi, x) d x .
$$


For $0=t_{1}<t_{2}$, using Fubini's theorem again, we get

$$
\begin{aligned}
\Delta_{I}^{X}(u) & =\int_{-\infty}^{0} h(\xi) d \xi-\int_{-\infty}^{X_{t_{2}}} \int_{-\infty}^{0} h(\xi) G_{0, t_{2}}^{X}(\xi, x) d \xi d x \\
& =\int_{-\infty}^{0} h(\xi) d \xi-\int_{-\infty}^{0} h(\xi) P_{\xi}\left[\tau_{\xi}^{X}>t_{2}\right] d \xi=\int_{-\infty}^{0} h(\xi) P_{\xi}\left[0 \leq \tau_{\xi}^{X} \leq t_{2}\right] d \xi
\end{aligned}
$$

For $0<t_{1}<t_{2}$, similarly,

$$
\begin{aligned}
\Delta_{I}^{X}(u) & =\int_{-\infty}^{X_{t_{1}}} \int_{-\infty}^{0} h(\xi) G_{0, t_{1}}^{X}(\xi, x) d \xi d x-\int_{-\infty}^{X_{t_{2}}} \int_{-\infty}^{0} h(\xi) G_{0, t_{2}}^{X}(\xi, x) d \xi d x \\
& =\int_{-\infty}^{0} h(\xi) P_{\xi}\left[t_{1}<\tau_{\xi}^{X} \leq t_{2}\right] d \xi
\end{aligned}
$$

Then for $I_{\epsilon}=\left[t_{1}-\epsilon, t_{1}\right]$, we get

$$
\begin{aligned}
\lim _{\epsilon \rightarrow 0} \Delta_{I_{\epsilon}}^{X}(u) & =\lim _{\epsilon \rightarrow 0}-\frac{1}{2} \int_{I_{\epsilon}} u_{x}\left(X_{t}^{-}, t\right) d t=0=\lim _{\epsilon \rightarrow 0} \int_{-\infty}^{0} h(\xi) P_{\xi}\left[t_{1}-\epsilon<\tau_{\xi}^{X} \leq t_{1}\right] d \xi \\
& =\int_{-\infty}^{0} h(\xi) P_{\xi}\left[\tau_{\xi}^{X}=t_{1}\right] d \xi .
\end{aligned}
$$

Finally we conclude that

$$
\Delta_{I}^{X}(u)=\int_{-\infty}^{0} h(\xi) P_{\xi}\left[t_{1} \leq \tau_{\xi}^{X} \leq t_{2}\right] d \xi=F_{h}^{X}(I) .
$$

By approximating the initial delta measure of Theorem 1.1, we prove the proposition below.

Proposition 3.2. Let $X$ be a Hölder continuous curve on any finite interval in $[0, \infty)$ with exponent $\gamma \in(1 / 2,1]$ and let $X_{0}=0$. We fix $r_{0}<X_{0}=0$ and choose $a$ sequence $\left\{h_{n}\right\} \subset C_{c}^{\infty}\left(\mathbb{R} ; \mathbb{R}_{+}\right)$with supp $h_{n}=\left[r_{0}-\frac{1}{n}, r_{0}+\frac{1}{n}\right] \Subset(-\infty, 0)$ and $\left\|h_{n}\right\|_{1}=$ 1. For each $h_{n}$, there exists a corresponding solution $u_{n}$ with $-\left.\frac{1}{2} \frac{\partial u_{n}}{\partial x}\right|_{\left(X_{t}^{-}, t\right)}=$ : $p_{n}(t)$ in the sense of Theorem 2.1. Then we have the following statements:

(1) There is a unique $p \in C([0, \infty))$ with $p(0)=0$ such that

$$
p(t)=-G_{x}\left(X_{t}, t ; r_{0}, 0\right)+\int_{0}^{t} G_{x}\left(X_{t}, t ; X_{\tau}, \tau\right) p(\tau) d \tau \text { for all } t>0
$$

(2) $p_{n}$ converges to $p$ in $C_{(\eta)}^{0}((0, T])$ for all $0<\eta<1 / 2$.

We use the following lemma extensively to prove Proposition 3.2:

Lemma 3.3. $\int_{0}^{t} \tau^{\alpha_{1}}(t-\tau)^{\alpha_{2}} d \tau=\frac{\Gamma\left(1+\alpha_{1}\right) \Gamma\left(1+\alpha_{2}\right)}{\Gamma\left(2+\alpha_{1}+\alpha_{2}\right)} t^{1+\alpha_{1}+\alpha_{2}}$ for $\alpha_{1}, \alpha_{2}>-1$, where $\Gamma$ is the gamma function. 
Proof of Proposition 3.2: Let $T_{s}>0$. Since $\left|G_{x}\left(X_{t}, t ; X_{\tau}, \tau\right)\right|=$ $\left|\frac{1}{\sqrt{2 \pi(t-\tau)}}\left\{-\frac{X_{t}-X_{\tau}}{t-\tau}\right\} \exp \left(-\frac{\left(X_{t}-X_{\tau}\right)^{2}}{2(t-\tau)}\right)\right| \leq \frac{C_{0}}{(t-\tau)^{\frac{3}{2}-\gamma}}$, we deduce that for $q_{1}, q_{2} \in$ $C\left(\left[0, T_{s}\right]\right), 0<t \leq T_{s}$,

$$
\begin{array}{r}
\left|\int_{0}^{t} G_{x}\left(X_{t}, t ; X_{\tau}, \tau\right)\left(q_{1}(\tau)-q_{2}(\tau)\right) d \tau\right| \leq \int_{0}^{t} \frac{C_{0}\left\|q_{1}-q_{2}\right\|_{T_{s}}}{(t-\tau)^{\frac{3}{2}-\gamma}} d \tau \\
=C_{1} t^{\gamma-\frac{1}{2}}\left\|q_{1}-q_{2}\right\|_{T_{s}} \leq C_{1} T_{s}^{\gamma-\frac{1}{2}}\left\|q_{1}-q_{2}\right\|_{T_{s}} .
\end{array}
$$

We define $F: C\left(\left[0, T_{s}\right]\right) \rightarrow C\left(\left[0, T_{s}\right]\right)$ as, for $q \in C\left(\left[0, T_{s}\right]\right)$,

$$
(F q)(t)=-G_{x}\left(X_{t}, t ; r_{0}, 0\right)+\int_{0}^{t} G_{x}\left(X_{t}, t ; X_{\tau}, \tau\right) q(\tau) d \tau \text { for } t>0,
$$

and $(F q)(0)=0$. Since $\lim _{t \rightarrow 0} G_{x}\left(X_{t}, t ; r_{0}, 0\right)=0$, it is well-defined. If we choose $T_{s}$ such that $C_{1} T_{s}^{\gamma-\frac{1}{2}}<1$, then $F$ is a contraction mapping so that $F$ has a unique fixed point since $C\left(\left[0, T_{s}\right]\right)$ is a Banach space. Let's call this $p_{T_{s}}$.

Now we assume that we have $p_{T_{s}}$ for some $T_{s}>0$. For $T^{\star}>T_{s}$, we define $H: C\left(\left[T_{s}, T^{\star}\right]\right) \rightarrow C\left(\left[T_{s}, T^{\star}\right]\right)$ as, for $q \in C\left(\left[T_{s}, T^{\star}\right]\right)$,

$$
\begin{aligned}
(H q)(t)= & -G_{x}\left(X_{t}, t ; r_{0}, 0\right)+\int_{0}^{T_{s}} G_{x}\left(X_{t}, t ; X_{\tau}, \tau\right) p_{T_{s}}(\tau) d \tau \\
& +\int_{T_{s}}^{t} G_{x}\left(X_{t}, t ; X_{\tau}, \tau\right) q(\tau) d \tau .
\end{aligned}
$$

Then for $q_{1}, q_{2} \in C\left(\left[T_{s}, T^{\star}\right]\right)$, we have

$$
\left\|H q_{1}-H q_{2}\right\|_{\infty} \leq C_{2}\left(t-T_{s}\right)^{\gamma-\frac{1}{2}}\left\|q_{1}-q_{2}\right\|_{\infty} \leq C_{2}\left(T^{\star}-T_{s}\right)^{\gamma-\frac{1}{2}}\left\|q_{1}-q_{2}\right\|_{\infty} .
$$

Similarly, if we choose $T^{\star}$ such that $C_{2}\left(T^{\star}-T_{s}\right)^{\gamma-\frac{1}{2}}<1$, then $H$ is a contraction mapping so that $H$ has a unique fixed point since $C\left(\left[T_{s}, T^{\star}\right]\right)$ is a Banach space.

Therefore, if we have $p$ defined $\left[0, T_{s}\right], p_{T_{s}}$, then we can extend this to time $T_{s}+C_{3}$ where $C_{3}$ is an independent constant. Thus if we repeat this step inductively, we have $p$ defined on $[0, \infty)$ which satisfies (3.11).

We now prove that $p_{n}$ converges to $p$ in $C_{(\eta)}^{0}\left(\left(0, T_{s}\right]\right)$ for all sufficiently small $T_{s}>0$. By (2.3),

$$
p_{n}(t)=-\int_{-\infty}^{0} h_{n}(\xi) G_{x}\left(X_{t}, t ; \xi, 0\right) d \xi+\int_{0}^{t} G_{x}\left(X_{t}, t ; X_{\tau}, \tau\right) p_{n}(\tau) d \tau .
$$

For $0<t \leq T_{s}$, taking the difference between (3.11) and (3.14), we get

$$
\begin{aligned}
t^{1-\eta}\left|p_{n}(t)-p(t)\right| & \leq t^{1-\eta}\left|\int_{-\infty}^{0} h_{n}(\xi)\left[G_{x}\left(X_{t}, t ; \xi, 0\right)-G_{x}\left(X_{t}, t ; r_{0}, 0\right)\right] d \xi\right| \\
& +t^{1-\eta}\left|\int_{0}^{t} G_{x}\left(X_{t}, t ; X_{\tau}, \tau\right)\left(p_{n}(\tau)-p(\tau)\right) d \tau\right|
\end{aligned}
$$

For the second term of the right hand side, we have

$$
\begin{array}{r}
t^{1-\eta}\left|\int_{0}^{t} G_{x}\left(X_{t}, t ; X_{\tau}, \tau\right)\left(p_{n}(\tau)-p(\tau)\right) d \tau\right| \leq C_{0} t^{1-\eta} \int_{0}^{t} \frac{\left\|p_{n}-p\right\|_{T_{s}}^{(\eta)}}{(t-\tau)^{\frac{3}{2}-\gamma} \tau^{1-\eta}} d \tau \\
=C_{1} t^{\gamma-\frac{1}{2}}\left\|p_{n}-p\right\|_{T_{s}}^{(\eta)} \leq C_{1} T_{s}^{\gamma-\frac{1}{2}}\left\|p_{n}-p\right\|_{T_{s}}^{(\eta)} .
\end{array}
$$


Let us choose $T_{s}>0$ such that $C_{1} T_{s}^{\gamma-\frac{1}{2}}<1$. Then

$$
\begin{gathered}
\left(1-C_{1} T_{s}^{\gamma-\frac{1}{2}}\right)\left\|p_{n}-p\right\|_{T_{s}}^{(\eta)} \leq t^{1-\eta}\left|\int_{-\infty}^{0} h_{n}(\xi)\left[G_{x}\left(X_{t}, t ; \xi, 0\right)-G_{x}\left(X_{t}, t ; r_{0}, 0\right)\right] d \xi\right| . \\
\leq \int_{-\infty}^{0} h_{n}(\xi) \sup _{\substack{0<t \leq T_{s} \\
\xi \in\left[r_{0}-\frac{1}{n}, r_{0}+\frac{1}{n}\right]}} t^{1-\eta}\left|G_{x}\left(X_{t}, t ; \xi, 0\right)-G_{x}\left(X_{t}, t ; r_{0}, 0\right)\right| d \xi .
\end{gathered}
$$

For all sufficiently large $n, 0<t \leq \frac{1}{n}$ and $r_{0}-\frac{1}{n} \leq \xi \leq r_{0}+\frac{1}{n}$, there exists $C_{2}$ such that

$$
\left|G_{x}\left(X_{t}, t ; \xi, 0\right)\right|=\left|\frac{1}{\sqrt{2 \pi}}\left\{-\frac{X_{t}-\xi}{t^{\frac{3}{2}}}\right\} \exp \left(-\frac{\left(X_{t}-\xi\right)^{2}}{2 t}\right)\right| \leq C_{2},
$$

Thus

$$
\sup _{\substack{0<t \leq \frac{1}{n} \\ \xi \in\left[r_{0}-\frac{1}{n}, r_{0}+\frac{1}{n}\right]}} t^{1-\eta}\left|G_{x}\left(X_{t}, t ; \xi, 0\right)-G_{x}\left(X_{t}, t ; r_{0}, 0\right)\right| \leq \sup _{0<t \leq \frac{1}{n}} 2 t^{1-\eta} C_{2} \leq \frac{2 C_{2}}{n^{1-\eta}} .
$$

For all sufficiently large $n, \frac{1}{n}<t \leq T_{s}$ and $r_{0}-\frac{1}{n} \leq \xi \leq r_{0}+\frac{1}{n}$, since $\left|G_{x x}(\cdot, t ; \cdot, 0)\right| \leq \frac{C_{3}}{t^{\frac{3}{2}}}$, we have

$$
\left|G_{x}\left(X_{t}, t ; \xi, 0\right)-G_{x}\left(X_{t}, t ; r_{0}, 0\right)\right| \leq \frac{C_{3}\left|\xi-r_{0}\right|}{t^{\frac{3}{2}}} \leq \frac{C_{3}}{n t^{\frac{3}{2}}}
$$

Thus

$$
\sup _{\substack{n \\ \frac{1}{n}<t \leq T_{s} \\ \xi \in\left[r_{0}-\frac{1}{n}, r_{0}+\frac{1}{n}\right]}} t^{1-\eta}\left|G_{x}\left(X_{t}, t ; \xi, 0\right)-G_{x}\left(X_{t}, t ; r_{0}, 0\right)\right| \leq \sup _{\frac{1}{n}<t \leq T_{s}} \frac{C_{3}}{n t^{\frac{1}{2}+\eta}} \leq \frac{C_{3}}{n^{\frac{1}{2}-\eta}} .
$$

Therefore, we conclude that $p_{n}$ converges to $p$ in $C_{(\eta)}^{0}\left(\left(0, T_{s}\right]\right)$ for all sufficiently small $T_{s}>0$.

To extend from $T_{s}$ to $T^{\star}$, assuming that $p_{n}$ converges to $p$ in $C_{(\eta)}^{0}\left(\left(0, T_{s}\right]\right)$ for some $T_{s}>0$ and writing $\left\|p_{n}-p\right\|_{\left[T_{s}, T^{\star}\right]}=\sup _{\tau \in\left[T_{s}, T^{\star}\right]}\left|p_{n}(\tau)-p(\tau)\right|$, for $T_{s} \leq t \leq T^{\star}$, we deduce that

$$
\begin{aligned}
& \left|p_{n}(t)-p(t)\right| \leq\left|\int_{-\infty}^{0} h_{n}(\xi)\left[G_{x}\left(X_{t}, t ; \xi, 0\right)-G_{x}\left(X_{t}, t ; r_{0}, 0\right)\right] d \xi\right| \\
& +\left|\int_{0}^{T_{s}} G_{x}\left(X_{t}, t ; X_{\tau}, \tau\right)\left(p_{n}(\tau)-p(\tau)\right) d \tau\right|+\left|\int_{T_{s}}^{t} G_{x}\left(X_{t}, t ; X_{\tau}, \tau\right)\left(p_{n}(\tau)-p(\tau)\right) d \tau\right| \\
& \leq C_{3} \int_{-\infty}^{0} h_{n}(\xi) \frac{\left|\xi-r_{0}\right|}{t^{\frac{3}{2}}} d \xi+C_{0} \int_{0}^{T_{s}} \frac{\left\|p_{n}-p\right\|_{T_{s}}^{(\eta)}}{(t-\tau)^{\frac{3}{2}-\gamma} \tau^{1-\eta}} d \tau+C_{0} \int_{T_{s}}^{t} \frac{\left\|p_{n}-p\right\|_{\left[T_{s}, T^{\star}\right]}}{(t-\tau)^{\frac{3}{2}-\gamma}} d \tau \\
& \leq \frac{C_{3}}{n t^{\frac{3}{2}}}+C_{0} \int_{0}^{T_{s}} \frac{\left\|p_{n}-p\right\|_{T_{s}}^{(\eta)}}{\left(T_{s}-\tau\right)^{\frac{3}{2}-\gamma} \tau^{1-\eta}} d \tau+C_{4}\left\|p_{n}-p\right\|_{\left[T_{s}, T^{\star}\right]}\left(t-T_{s}\right)^{\gamma-\frac{1}{2}} \\
& \leq \frac{C_{3}}{n T_{s}^{\frac{3}{2}}}+C_{1} T_{s}^{\gamma-\frac{3}{2}+\eta}\left\|p_{n}-p\right\|_{T_{s}}^{(\eta)}+C_{4}\left\|p_{n}-p\right\|_{\left[T_{s}, T^{\star}\right]}\left(T^{\star}-T_{s}\right)^{\gamma-\frac{1}{2}} .
\end{aligned}
$$


Let us choose $T^{\star}>T_{s}$ such that $C_{4}\left(T^{\star}-T_{s}\right)^{\gamma-\frac{1}{2}}<1$, then we have

$$
\left(1-C_{4}\left(T^{\star}-T_{s}\right)^{\gamma-\frac{1}{2}}\right)\left\|p_{n}-p\right\|_{\left[T_{s}, T^{\star}\right]} \leq \frac{C_{3}}{n T_{s}^{\frac{3}{2}}}+C_{1} T_{s}^{\gamma-\frac{3}{2}+\eta}\left\|p_{n}-p\right\|_{T_{s}}^{(\eta)} .
$$

The right term vanishes when $n$ goes to $\infty$ so that $p_{n}$ converges to $p$ in $C_{(\eta)}^{0}\left(\left(0, T_{s}+\right.\right.$ $\left.C_{5}\right]$ ) for some independent constant $C_{5}>0$. By repeating this argument inductively, it follows that $p_{n}$ converges to $p$ in $C_{(\eta)}^{0}((0, T])$.

Now we can prove $p$ from Proposition 3.2 is the density function of $F_{r_{0}}^{X}$. Hence we have

$$
\lim _{n \rightarrow \infty} \int_{-\infty}^{0} h_{n}(\xi) P_{\xi}\left(\tau_{\xi}^{X} \in I\right) d \xi=\lim _{n \rightarrow \infty} \int_{I} p_{n}(t) d t=\int_{I} p(t) d t .
$$

For $I=[0, t] \subset[0, T], P_{\xi}\left(\tau_{\xi}^{X} \in I\right)$ is an increasing function of $\xi$, so if we choose $h_{n}$ so that $\lim _{n \rightarrow \infty} \int_{r_{0}}^{r_{0}+\frac{1}{n}} h_{n}(\xi) d \xi=1$, we get

$\lim _{\xi \rightarrow r_{0}^{+}} P_{\xi}\left(\tau_{\xi}^{X} \in I\right) \leq \lim _{n \rightarrow \infty} \int_{r_{0}}^{r_{0}+\frac{1}{n}} h_{n}(\xi) P_{\xi}\left(\tau_{\xi}^{X} \in I\right) d \xi=\lim _{n \rightarrow \infty} \int_{-\infty}^{0} h_{n}(\xi) P_{\xi}\left(\tau_{\xi}^{X} \in I\right) d \xi$.

Similarly, if we choose $h_{n}$ so that $\lim _{n \rightarrow \infty} \int_{r_{0}-\frac{1}{n}}^{r_{0}} h_{n}(\xi) d \xi=1$, we get

$$
\lim _{n \rightarrow \infty} \int_{-\infty}^{0} h_{n}(\xi) P_{\xi}\left(\tau_{\xi}^{X} \in I\right) d \xi=\lim _{n \rightarrow \infty} \int_{r_{0}-\frac{1}{n}}^{r_{0}} h_{n}(\xi) P_{\xi}\left(\tau_{\xi}^{X} \in I\right) d \xi \leq \lim _{\xi \rightarrow r_{0}^{-}} P_{\xi}\left(\tau_{\xi}^{X} \in I\right) .
$$

Therefore, we obtain that

$$
F_{r_{0}}^{X}([0, t])=P_{r_{0}}\left(\tau_{r_{0}}^{X} \leq t\right)=\int_{0}^{t} p(\tau) d \tau,
$$

which implies that $p$ is the density function of $F_{r_{0}}^{X}$, thus item 2 is proved.

By Theorem 2.1 and the properties of the Gaussian kernel, $G_{0, t}^{X}\left(r_{0}, x\right)$ solves (1.4), (1.5) and (1.6). Hence $G^{X}$ is the Green function of the heat equation with Dirichlet boundary condition which implies item 4. Furthermore, $G_{0, t}^{X}\left(r_{0}, x\right)$ can be written as

$$
G_{0, t}^{X}\left(r_{0}, x\right)=G_{0, t}\left(r_{0}, x\right)-\int_{0}^{t} G_{\tau, t}\left(X_{\tau}, x\right) p(\tau) d \tau .
$$

Differentiating with respect to $\mathrm{x}$, applying the jump relation and (3.11), we have

$$
\begin{aligned}
\left.\frac{\partial}{\partial x} G_{0, t}^{X}\left(r_{0}, x\right)\right|_{x=X_{t}^{-}} & =\frac{\partial}{\partial x} G_{0, t}\left(r_{0}, X_{t}\right)-p(t)-\int_{0}^{t} \frac{\partial}{\partial x} G_{\tau, t}\left(X_{\tau}, X_{t}\right) p(\tau) d \tau \\
& =-2 p(t)
\end{aligned}
$$

Thus $p(t)=-\left.\frac{1}{2} \frac{\partial}{\partial x} G_{0, t}^{X}\left(r_{0}, x\right)\right|_{x=X_{t}^{-}}$, so it proves item 3 of Theorem 1.1.

\section{Acknowledgements}

I am thankful to Errico Presutti, Anna De Masi for helpful suggestions and Victor Perez Abreu, an anonymous referee for useful comments. 


\section{References}

L. Alili and P. Patie. Boundary crossing identities for Brownian motion and some nonlinear ODE's. Proc. Amer. Math. Soc. 142 (11), 3811-3824 (2014). MR3251722.

K. Borovkov and A. N. Downes. On boundary crossing probabilities for diffusion processes. Stochastic Process. Appl. 120 (2), 105-129 (2010). MR2576883.

J. R. Cannon. The one-dimensional heat equation, volume 23 of Encyclopedia of Mathematics and its Applications. Addison-Wesley Publishing Company, Advanced Book Program, Reading, MA (1984). ISBN 0-201-13522-1. MR747979.

G. Carinci, A. De Masi, C. Giardinà and E. Presutti. Free boundary problems in PDEs and particle systems, volume 12 of SpringerBriefs in Mathematical Physics. Springer, [Cham] (2016). ISBN 978-3-319-33369-4; 978-3-319-333700. MR3497333.

A. Fasano. Mathematical models of some diffusive processes with free boundaries, volume 11 of MAT. Serie A: Conferencias, Seminarios y Trabajos de Matemática [MAT. Series A: Mathematical Conferences, Seminars and Papers]. Universidad Austral, Facultad de Ciencias Empresariales (FCE-UA), Departamento de Matemática, Rosario (2005). MR2285006.

I. Karatzas and S. E. Shreve. Brownian motion and stochastic calculus, volume 113 of Graduate Texts in Mathematics. Springer-Verlag, New York, second edition (1991). ISBN 0-387-97655-8. MR1121940.

G. Peskir and A. Shiryaev. Optimal stopping and free-boundary problems. Lectures in Mathematics ETH Zürich. Birkhäuser Verlag, Basel (2006). ISBN 978-3-76432419-3; 3-7643-2419-8. MR2256030.

L. M. Ricciardi, L. Sacerdote and S. Sato. On an integral equation for first-passagetime probability densities. J. Appl. Probab. 21 (2), 302-314 (1984). MR741132.

T. Taillefumier and M. Magnasco. A transition to sharp timing in stochastic leaky integrate-and-fire neurons driven by frozen noisy input. Neural Comput. 26 (5), 819-859 (2014). MR3221038. 